\title{
Patent Foramen Ovale- \\ Its Correlation with Other Maladies and a Review of Detection Screening
}

\author{
Michael W Jopling, MD¹, John A Kurowski, BS, RN² and Suzanne M Williams, MS² \\ 1. Chief Medical Officer, Cardiox Corp, Columbus, Ohio, US; 2. Clinical Educator, Cardiox Corp, Columbus, Ohio, US
}

\begin{abstract}
Patent foramen ovale (PFO) is a condition that often remains undiagnosed despite studies showing it has a prevalence as high as $25 \%$ in the adult population. Blood passing from the right to the left atria across a PFO bypasses the pulmonary circulation allowing embolic debris, deoxygenated blood, and various vasoactive substances direct access to the systemic circulation. Studies suggest that a PFO may contribute to certain illnesses such as: cryptogenic stroke, decompression illness, venous air embolism, migraine with aura, and others due to a higher prevalence in patients with these problems than in the general population. Knowledge of the presence of a PFO may become an important aspect in the clinical management of disease. Various technology tools that are utilized to assess circulatory right-to-left shunt abnormalities, such as cardiac ultrasound visualization, transcranial Doppler, and dye dilution curves, are reviewed as well as the importance of the Valsalva procedure in creating a positive right-to-left atrial pressure gradient.
\end{abstract}

\section{Keywords}

Patent foramen ovale, right-to-left shunt, Valsalva, dye dilution curves, transient ischemic attack, transcranial Doppler, transesophageal echo, transthoracic echo, bubble study, paradoxical embolism, cryptogenic stroke

Disclosure: Michael W Jopling, MD, John A Kurowski, BS, RN, and Suzanne M Williams, MS, report personal fees from Cardiox Corp during the conduct of the study and personal fees from Cardiox Corp outside the submitted work.

Open Access: This article is published under the Creative Commons Attribution Noncommercial License, which permits any noncommercial use, distribution, adaptation,

and reproduction provided the original author(s) and source are given appropriate credit.

Received: July 31, 2015 Accepted: September 3, 2015 Citation: US Neurology 2015;11(2):89-95 DOl: http://doi.org/10.17925/USN.2015.11.02.89

Correspondence: Michael W Jopling, MD, 4100 Horizons Drive, Suite 100, Columbus, OH 43320, US. E: michaeljopling@cardiox.com

Support: The publication of this article was supported by Cardiox Corp. The views and opinions expressed are those of the authors and not necessarily those of

Cardiox Corp.

Patent foramen ovale (PFO) is an opening or flap in the atrial septum that exists as a normal and vital component of intrauterine life. PFO allows for the transportation of rich oxygenated blood to bypass the lungs and flow from the right to the left atrium during fetal development. Shortly after birth, a reduction in right heart pressure related to a decrease in resistance through the newly functioning respiratory system closes the flap. However, sometimes a PFO fails to close properly after birth.

There are no known risk factors for PFO, but it is found present in approximately one-quarter of the adult population. ${ }^{1}$ PFO usually has no signs or symptoms and typically goes undiagnosed in the majority of people. The amount of blood that passes through a PFO is small; however, it can permit arterial circulation, blood clots, and vasoactive substances in the venous system to bypass the lungs and be directly introduced into the oxygen-rich blood, which in turn can manifest clinically. Mayo Clinic autopsy studies revealed that the size of the PFO increases from a mean of $3.4 \mathrm{~mm}$ in the first decade of life to $5.8 \mathrm{~mm}$ in the tenth decade of life. ${ }^{2}$ Knowing the status of a PFO and how it relates to other medical conditions may be important in disease management.

Commonly neurologists become involved early in the evaluation of clinical manifestations of various processes that are the result of the presence of PFO. This manuscript aims to assist the neurologist in his or her interactions with other consultants and to provide a high-level update of knowledge regarding the various associated conditions and an overview of diagnostic methods.

\section{Clinical Significance}

Studies have shown that PFO is linked to increased risk for several pathologic processes, such as paradoxical embolism in cryptogenic stroke, venous-to-arterial gas embolism in serious forms of decompression illness (DCI), along with many other clinical implications. In addition, it is often associated with atrial septal aneurysm and Chiari network, although these variations are uncommon. ${ }^{3}$ Studies have also demonstrated that the size of a PFO affects the risk for stroke. Mean PFO diameter was significantly 
larger $(4 \pm 2 \mathrm{~mm}$ ) in patients with cryptogenic cerebrovascular events than in controls $(2 \pm 1 \mathrm{~mm})$ or in patients with strokes of a defined cause. ${ }^{4}$

\section{Patent Foramen Ovale and Stroke}

According to the World Health Organization (WHO), 15 million people suffer stroke worldwide each year. Of these, five million die while another five million are left permanently disabled. In the US, stroke is the third leading cause of death -with approximately 600,000 people experiencing stroke for the first time and an additional 185,000 experiencing recurrent stroke. As much as $40 \%$ of these strokes cannot be identified as having any formative cause or factor, and therefore fall into the cryptogenic stroke category.

Even though the majority of ischemic strokes (85\%) occur in persons older than 65 years of age in tandem with development of atherosclerosis, a minority of ischemic strokes affects younger patients, to which as much as $43 \%$ are cryptogenic. The most prevalent potential source of cardioembolism in young adults with cryptogenic stroke is PFO, which is detected in more than half of such persons. ${ }^{5}$ Although there is a high prevalence of PFOs in young patients with cryptogenic stroke, Dr Kim and Dr Saver suggest that PFO itself is not necessarily a prediction of stroke risk. They state that young patients with the strongest potential for stroke are those who have PFO and atrial septal aneurysm. The risk for stroke in these patients is about 33 times higher than just PFO alone. Additional risks determined to affect the potential for stroke in young people include the size of PFO and the degree of functional shuntingparticularly patients with PFOs have greater right-to-left shunting (RLS) at rest compared with controls. The degree of RLS is related to not only PFO size, but also the presence of a prominent Eustachian valve that directs blood toward the PFO from the inferior vena. ${ }^{6}$

The link between PFO and stroke remains evident as it continues to be researched. In 2013, a team studied randomized controlled trials that compared PFO device closure against medical therapy. They took three eligible studies (CLOSURE 1, RESPECT, PC TRIAL) and pooled the results according to the patients' populations: intention-to-treat, per protocol, and as-treated cohorts. A supplementary subset analysis of RESPECT and PC TRIAL pooled only their results as they utilized the same closure device. The results yielded effect-estimate hazard ratios of $0.67 \%$ in the intention-to-treat cohort, $0.62 \%$ in the per-protocol cohort, and $0.61 \%$ in the as-treated cohort, showing beneficial effects of device closure. The results, when pooled with the additional RESPECT and PC TRIAL, showed an effect-estimate hazard ratio of $0.54,0.48$, and $0.42 \%$, respectively. The primary end point of this study failed to show a significant benefit to PFO closure an adults with cryptogenic stroke, yet they concluded that PFO closure was more beneficial than medical therapy in the prevention of recurrent neurological events. ${ }^{7}$ These data further support the role PFO has in the increased risk for stroke and the importance of closure once detected.

The Risk of Paradoxical Embolism (RoPE) meta-analysis study created an 11-point scoring system where 1 point (each factor) is given if a patient lacks a history of hypertension, diabetes, stroke/TIA or is a nonsmoker, or has a cortical infarct on imaging. These factors therefore can combine for a score ranging from 0 to 5 . In addition, points are added based upon age: 18-29 (5 points); 30-39 (4 points); 40-49 (3 points); 50-59 (2 points); 60-69 (1 point); >70 (0 point). Using this scoring system the
PFO prevalence was $23 \%$ if the RoPE score was 0-3 and increased linearly to a PFO prevalence of $73 \%$ if the RoPE score was 9-10. This scoring system has been useful in estimating the general PFO prevalence in a population, which can become useful in determining the patient selection for potential PFO closure. ${ }^{8}$

\section{Patent Foramen Ovale and Migraine}

The relationship between migraine and PFO continues to undergo research. Although data have yielded mixed results, there appears to be some evidence that PFO affects the presence of migraine when accompanied by aura. A study has shown that those who suffer migraine with an aura beforehand were twice as likely to have a PFO (41-48 \%) than the general population. ${ }^{9}$ A 2003 study assessed the prevalence of migraine in a large patient population with a presumed PFO-mediated paradoxical embolism. The results, derived between 1998 and 2002, of 215 subjects had a demonstrated prevalence of headache of $33 \%$ prior to PFO closure, which was reduced by $54 \%$ in patients with migraine with aura, and by $62 \%$ in patients with migraine without aura. ${ }^{10}$ The study demonstrated that patients with a presumed PFO were twice as likely to have migraine with aura over the general population and that PFO closure reduced the frequency of migraine attacks both with and without aura.

Reisman, in 2005, investigated the effects of transcatheter closure on migraine frequency in patients with paradoxical embolism and pointed to a probable link between PFO and migraine attacks. At the time of closure, 57 of 162 (35\%) of participants experienced migrai ne symptoms; 39 of 57 (68\%) experienced migraine with aura; and seven of the 57 (14 \%) reported a significant reduction in migraine frequency. ${ }^{11}$ Reisman reported an 80 \%reduction in the mean number of migraine episodes per month after PFO closure.

However, a more recent study, conducted by St Jude in its third randomized trial (PREMIUM) in 2015, raises some questions regarding the legitimacy of PFO closure as a treatment for patients with migraine. The study investigated whether or not implantation of the Amplatzer ${ }^{\mathrm{TM}}$ Septal Occluder (AGA Medical Corporation, Golden valley, MN) would help relieve symptoms of migraine in patients with a known PFO. In the blind study, 117 patients received the closure device, while 103 received a sham procedure and medical management. Unfortunately, the study group did not reach its primary end point of obtaining a $50 \%$ reduction in migraine frequency. However, even though the primary goal was not accomplished, some secondary end points did still achieve significance. Of all the patients, $8.5 \%$, of which $11 \%$ had migraines accompanied by aura, had complete migraine remission. ${ }^{12}$

Although an established pathway has not been identified between PFO and migraine, it appears that in some cases, especially those where migraine includes aura, PFO closure may positively affect frequency and duration.

\section{Patent Foramen Ovale and Sleep Apnea}

Obstructive sleep apnea (OSA) is a common disorder affecting $24 \%$ and $9 \%$ of middle-aged men and women. OSA constitutes a major public health concern because of its prevalence among cardiovascular complications such as hypertension, ischemic heart disease, heart failure, stroke, pulmonary hypertension, and a variety of cardiac arrhythmias. ${ }^{13}$ 
Multiple studies have confirmed a plausible relationship between PFO and the occurrence of sleep apnea as causation for these cardiovascular events. Johansson et al. concluded that oxygen desaturations occurred more often, in proportion to the frequency of respiratory disturbances in OSA subjects with PFO than those without.

The study included a group of 209 patients diagnosed with OSA, whose proportion of desaturation to respiratory events was calculated as the ratio of oxygen desaturation index (ODI) to apnea-hypopnea index (AHI). A total of 15 cases of high proportional desaturation were individually matched with 15 controls with low proportional desaturation, all with pulmonary disease. PFO was assessed using transesophageal echocardiography (TEE) and considered large when $\geq 20$ bubbles passed from the right to the left atrium after a single injection.

The prevalence of a large PFO was nine out of $15(60 \%)$ in the highproportional desaturation group versus two out of 15 (13\%) in the lowproportional desaturation group. The median number of passing bubbles was positively correlated to minimum oxygen saturation among those with PFO. ${ }^{14}$

\section{Patent Foramen Ovale and Decompression Illness}

As many as 1,000 divers each year suffer DCl. Those who have PFO may be at an elevated risk for experiencing such an event after diving.

During normal decompression, nitrogen gas is released from peripheral tissues and absorbed by the body and lungs. However, if pressure changes too quickly, bubbles can form, causing painful and sometimes fatal symptoms. To equalize pressure in the middle ear, a diver is taught to frequently blow outward with a closed mouth and nose-commonly called a Valsalva maneuver. This simple act of exhaling opens a PFO and allows gas bubbles not dissolved in the blood to enter arterial circulation and cause a potential cerebral event.

Torti et al., in 2004, evaluated 230 divers to determine the absolute and relative odds of a $\mathrm{DCl}$ event, in relation to the presence and size of a PFO. The absolute risk for suffering a $\mathrm{DCl}$ event was determined as 2.5 per 1,000 . The study found 63 of the 230 (27 \%) divers had a PFO. Of the 63 divers, 18 (29 \%) divers with known PFO experienced a major DCl event, compared with only 10 of 167 (6\%) cases of major DCl events in divers without a PFO. Torti et al. concluded that the presence of a TEE-detected PFO was related to an absolute risk for five major DCl events per 10,000; five times higher than those without a PFO. ${ }^{15}$

\section{Patent Foramen Ovale and Dementia}

In the US alone, 4.5 million people have been diagnosed with some degree of dementia. Purandare found that spontaneous cerebral emboli was present in $40 \%$ of patients with Alzheimer's and nearly $37 \%$ of Alzheimer's have a PFO. ${ }^{16}$ Purandare also explored the role of paradoxical embolization in dementia by investigating cerebral emboli, venous to arterial circulation shunts, and carotid artery disease. The results of the study showed that cerebral emboli were detected in $27 \%$ of dementia patients compared with $7 \%$ in the control group. In the dementia patients with cerebral emboli, a venous to arterial shunt was detected in $64 \%$ and moderate to severe carotid stenosis was present in $30 \%{ }^{17}$ These data confirm paradoxical embolization as a potential mechanism for cerebral damage, influencing vascular dementia and Alzheimer's disease.

\section{Patent Foramen Ovale and Implantables}

There are over 5 million pacemakers and other cardiac implantable devices in the US. Each device carries an increased risk for creating and releasing emboli into the circulatory pathway. Thrombi accumulation caused by the leads may transfer via a PFO into arterial circulation, thereby introducing a greater chance of stroke or trans ischemic attack (TIA).

Desimone et al. looked at patients with endocardial lead implants with a primary end point of stroke/TIA that was consistent for a cardio embolic etiology and a secondary end point of mortality. The post implant period compared PFO versus non-PFO patients using the Cox proportional hazard models (adjusting for age, sex, previous history of stroke/ TIA, history of atrial fibrillation, and the use of aspirin, warfarin at the time of implant).

Investigators concluded that the presence of a PFO on routine echocardiography was associated with a substantial increased risk for embolic stroke/TIA. They further affirm the importance of screening for PFOs in patients who require cardiac implantable electronic devices, stating that if a PFO is detected, PFO closure, anticoagulation, or nonvascular lead placement may be considered. ${ }^{18}$

\section{Patent Foramen Ovale and Surgical Procedures}

PFO may increase the risk for embolism associated with total hip replacement (THR), as well as other surgical procedures.

A 2009 case report revealed that preoperative atrial septal defect (ASD occlusion could provide protection against paradoxical embolism during THR. ${ }^{19}$ In the case study, a 74-year-old patient was evaluated prior to an elective right hip replacement. The patient reported two consecutive cerebellar ischemic strokes in 2001, which revealed interatrial septum aneurysm with a PFO, and massive shunting with complete opacification of the left atrium. The patient eventually recovered, and it was decided that PFO closure was not necessary at the time.

Considering the high risk for venous embolism associated with THR, along with the patient's history, preoperative transcatheter closure of the PFO was recommended and performed prior to the THR. Echocardiography three months later revealed only minor shunting ( $<10$ bubbles) during a Valsalva. The hip surgery was carried out successfully with only aspirin therapy, and the patient suffered no neurologic incidence or ischemic problems in the postoperative period.

Venous air embolism (VAE) is no longer attributed to just neurosurgical procedures conducted in the "sitting position." It is now frequently encountered in broad areas such as interventional radiology, cardiac orthopedic transplant, left ventricular assist device implantation, or laparoscopic surgical centers-nearly any procedure involving anesthesia.

The prominence of PFO as a contributing factor in the occurrence of VAE was discussed by Mirski et al. who reported that PFO has been found to permit air directly into cerebral circulation and that patients with documented PFO appear to be at increased risk for paradoxical embolus during surgery. ${ }^{20}$ They also pointed out that early detection of PFO is the best prevention of such an event. 
Figure 1: Interaction of Venous Capacitance, RA Size, LA Pressure, RA Pressure, and Airway Pressure During a Valsalva ${ }^{31}$

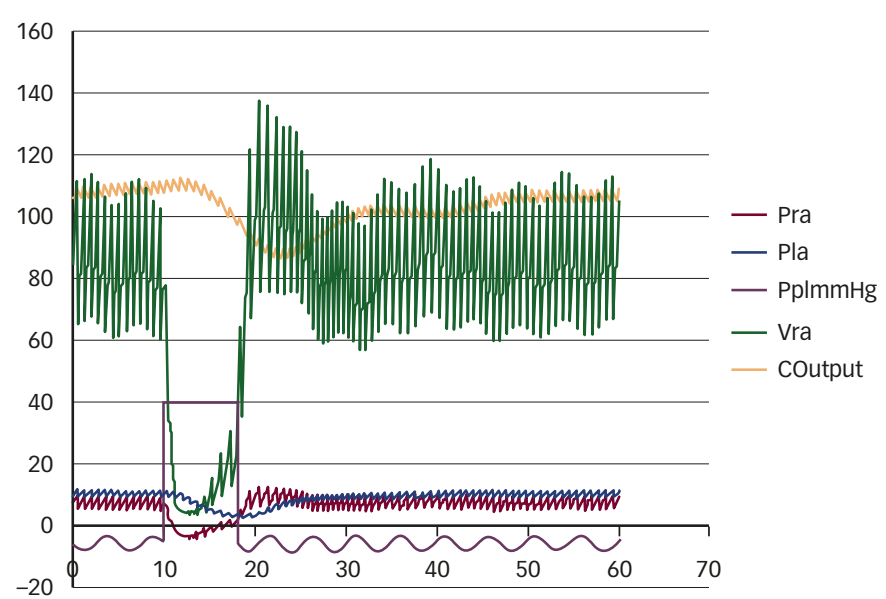

COutput = cardiac output; $L A=$ left atrial; $P l a=$ left atrial pressure; $P$ plmmHg = pleural pressure; Pra = right atrial pressure; $R A=$ right atrial; $V r a=$ right atrial volume.

\section{Figure 2: Detailed View Showing Reversal of Interatrial Pressure Gradient Immediately Following Release of Valsalva ${ }^{31}$}

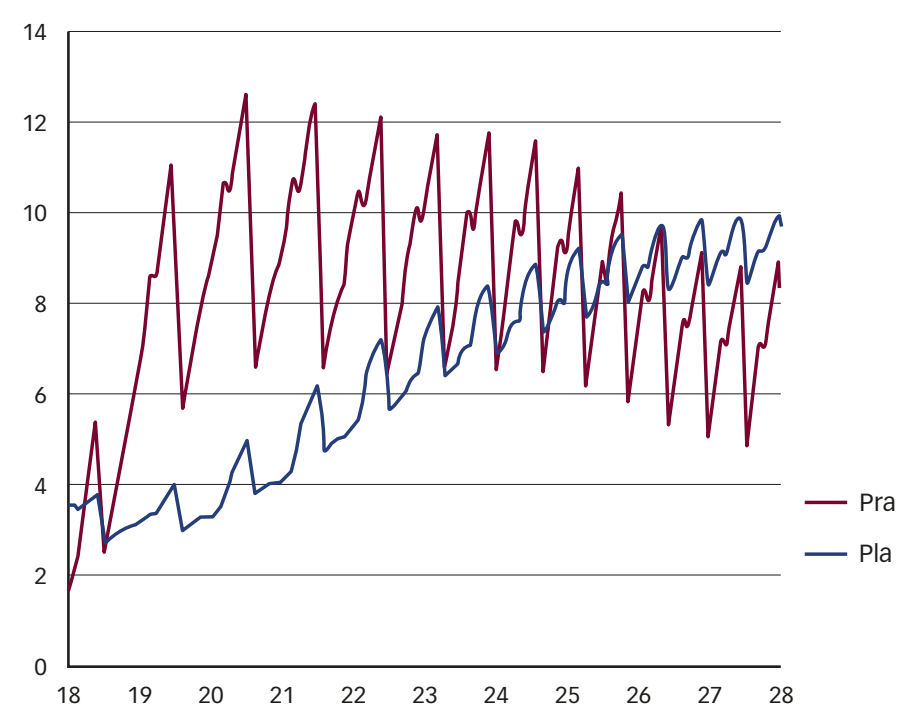

Pla = left atrial pressure; Pra = right atrial pressure.

\section{Testing Methods}

Currently there are several accepted practices being utilized in PFO detection, yet most of them were not specifically designed for such. consequently, there is widespread variance in confidence levels among the results presented in some of these methods. Limitations in sensitivity, specificity, and quantification pose the biggest concerns that need to be addressed.

\section{Transesophageal Echocardiography}

TEE is considered the "gold standard" in screening because it is perceived to be the superior method of testing. TEE is an invasive procedure that shows 2D ultrasound imaging of the heart from a transesophageal approach. It displays the anatomy and (with great variability from patient to patient), structural changes relating to septal defects such as PFO and ASD. Sharma et al. explains that although TEE is considered the gold standard, it is not always well tolerated by patients, requires an expert echocardiographer to read the results, and the Valsalva maneuver is impaired by sedation and the endoscope in the throat. ${ }^{21}$

A recent study identified TEE as significantly lacking sensitivity and specificity in diagnosing PFO. Mojadidi et al. found that TEE had a weighted sensitivity of $89.2 \%$ and specificity of $91.4 \%$ compared with cardiac surgery, catheterization, or autopsy. The study concluded that PFOs may still be missed altogether or misdiagnosed in TEE screening and that understanding these limitations should be evaluated before scheduling a patient for PFO closure. ${ }^{22}$

\section{Transthoracic Echo}

Transthoracic echo (TTE) is frequently used as a "first line" method of PFO assessment due to its economic value, and it is minimally invasive. An ultrasound probe is placed over the patient's chest, and like TEE, visualizes the heart's anatomy. This imaging is performed in conjunction with a contrast intravenous injection-agitated saline (bubble study). Since the patient does not require sedation, it is possible to obtain a voluntary valsalva maneuver. However, obesity, chronic lung disease, and even the Valsalva maneuver itself often present significant obstacles in obtaining adequate visualization of the atria through the chest wall due to the depth or the presence of lung tissue.

Even though TEE is the most popular of all three screening methods, it has the lowest sensitivity. One study compared 19 known patients with a known PFO. The TEE was only able to detect nine out of 19 (47\%) patients. The study concluded that the sensitivity of transthoracic echocardiography is heavily hampered by the frequency of inadequate heart visualization. ${ }^{23}$

\section{Transcranial Doppler}

Transcranial Doppler (TCD) is an alternative method for indirectly diagnosing intracardiac RLS by detecting air or contrast microbubbles passing through the intracranial arteries, with insonation of at least one middle cerebral artery (MCA). Shunt conductance is graded by a logarithmic scale (1-3) with 3 being used as a cutoff for a positive study.

Even though TCD has been shown to have high sensitivity and is more comfortable for the patient, TCD cannot localize the site of the RLS or determine its size. Therefore, TCD is recommended as a complementary procedure to TEE, to increase diagnostic accuracy in detecting PFO but not as a stand-alone procedure..$^{24}$

\section{Ear Oximetry}

First suggested by Karltunen et al., ${ }^{25}$ pulse oximetry $\left(\mathrm{SpO}_{2}\right)$ via an ear sensor has been utilized for RLS screening through the detection of an oxygen desaturation response following repetitive valsalva procedures. In a recent study of 165 scuba divers utilizing this technique with a threshold of $>0.825 \mathrm{SpO}_{2}$ change resulted in a sensitivity of 0.756 and a specificity of $0.706 .{ }^{26}$ This technique has the potential for use as a screening tool for large populations at a very low cost. $\mathrm{SpO}_{2}$, device time averaging, motion artifact, and venous blood induced changes in $\mathrm{SpO}_{2}$ may limit further improvement on these detection parameters. 
A study conducted in Finland, 2001, determined that there was an identifiable need for a low-cost, noninvasive method for detecting PFO. The study compared the accuracy of dye dilution curves with oximetry against contrast TEE. They concluded that dye dilution and oximetry are both sensitive and specific methods of detecting RLS. Furthermore, oximetry was primarily advantageous over the current diagnostic methods as it was noninvasive, safe, and inexpensive with no discomfort to the patient. The team suggested that oximetry could be used as a first-line screening method for PFO in patients with cryptogenic stroke. ${ }^{27}$

\section{Dye Dilution Curves}

Indicator dye dilution curves and ear oximetry were first used in the late 1950s when Carter, Swan, and Wood injected Evans blue dye into the superior vena cava and pulmonary arteries to determine that time and concentration were critical values in determining abnormal dilution curves. ${ }^{28}$

Throughout the 60s and 70s, studies continued to emerge that analyzed the use of dye dilution curves. One such team from Harvard Medical School and Brigham's Hospital in Boston, Massachusetts, employed a technique for detecting small defects of the atrial septum. They used a Valsalva maneuver to cause the right atrial (RA) pressure to exceed the left, forcing a transient RLS across the defect. They showed that the RLS was detected by an early appearance of deflection in the standard indicator dilution curves performed when injecting Indocyanine green dye (ICG) into the inferior vena cava during the critical Valsalva maneuver. ${ }^{29}$

In 1974, Victorica and Gessner presented a method for quantitating leftto-right shunts from dilution curves and the injection of an indicator (ICG) centrally in the right ventricle or pulmonary artery. They showed that blood was withdrawn rapidly from the aorta or central artery in the presence of a left-to-right shunt and that the recorded indicator curve displayed two peaks during such events. The formula they used to assess abnormal shunting has been proven a reliable estimate of the magnitude of the shunt expressed as a percent of pulmonary blood flow. ${ }^{30}$

These studies and many more have revealed that indicator dye dilution curves derived from ICG and a proper valsalva maneuver provide sensitive and reliable data in the detection of right-to-left cardiac shunts. The importance of the Valsalva maneuver when detecting cardiac flow abnormalities remains a critical component in PFO detection. The hemodynamic changes that occur during and after the forced expiration of the Valsalva maneuver are well documented.

Indicator Dye Dilution Curves in conjunction with a controlled Valsalva technology has been introduced to identify RLS via dye dilution curves sensed via fluorescence at a detection site on the ear.

\section{The Importance of a Valsalva Maneuver}

A valsalva maneuver consists of creating an increased intrathoracic pressure via use of the diaphragm and lungs while maintaining an open glottis. Simple blowing into a manometric device with a closed glottis using air only in the pharynx and cheeks will not be effective in creating an increase in intrathoracic pressure. ${ }^{31}$

The results of a Valsalva simulation (see Figure 1) 131 $^{31}$ llows us to inspect the complex interaction of venous capacitance, RA size, left atrial (LA)

\section{Table 1: Grading Scales of Braun and Windecker with Ultrasound Image Used in TEE and TTE Methods ${ }^{35,36}$}

\section{Scale Name}

\begin{tabular}{llll}
\multirow{2}{*}{ Braun } & Small & Medium & Large \\
& $3-10$ bubbles & 0-20 bubbles & $>20$ bubbles \\
Windecker & Grade I & Grade II & Grade III \\
& Minimal & Moderate & Severe \\
& $1-5$ bubbles & $6-20$ bubbles & $>20$ bubbles
\end{tabular}

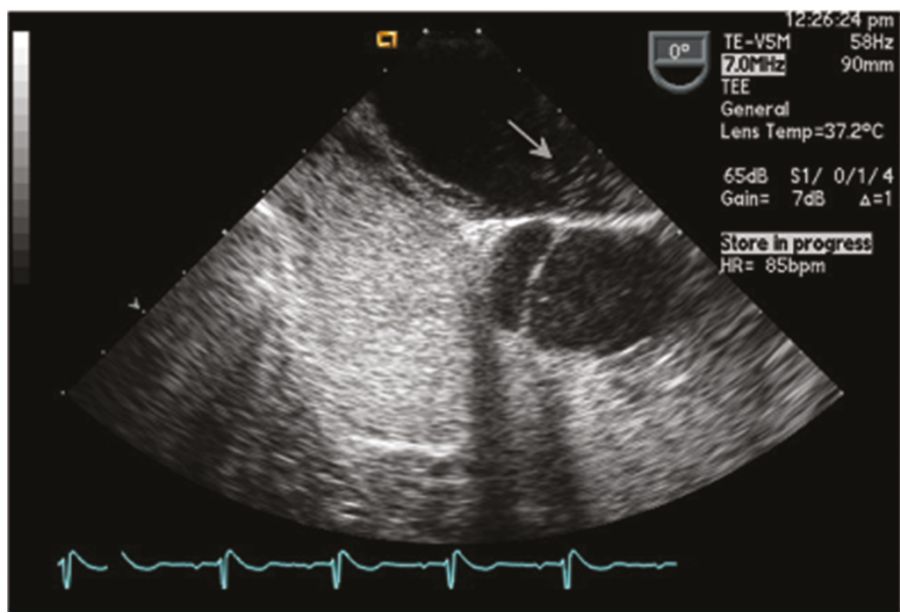

$T E E=$ transesophageal echocardiography; $T T E=$ transthoracic echo.

\section{Table 2: Spencer Algorithm Grading Scale with Sonography Images Used in TCD Method ${ }^{37}$}

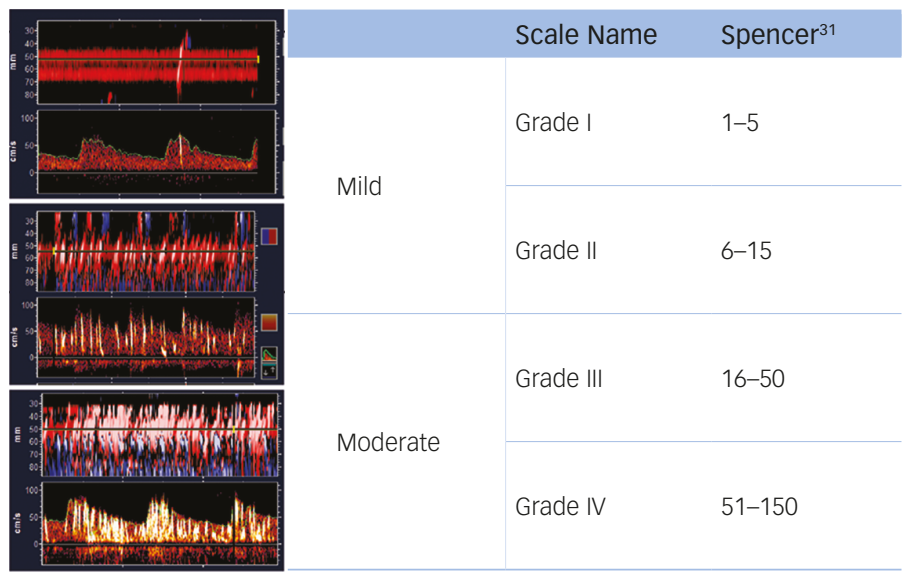

$T C D=$ transcranial Doppler

pressure, and RA pressure with the airway pressure. In the detailed view (see Figure 2) ${ }^{31}$ note the reversal of the interatrial pressure gradient that exists for only five cardiac cycles following the release of a Valsalva. An increase in intrathoracic pressure is necessary to impair venous return to the heart, and a significant decrease in the RA and LA volume occurs. Following the release of the Valsalva, venous return to the RA is first augmented due to blood that had been building up in the larger extrathoracic veins. LA volume return is delayed secondary to the longer route via the pulmonary circulation. Only during the time period when RA pressure is greater than LA pressure will a PFO open and a RLS occur. 


\section{Figure 3: Test Results Showing Two Peak Positive Shunt and One Peak Negative Shunt ${ }^{33}$}
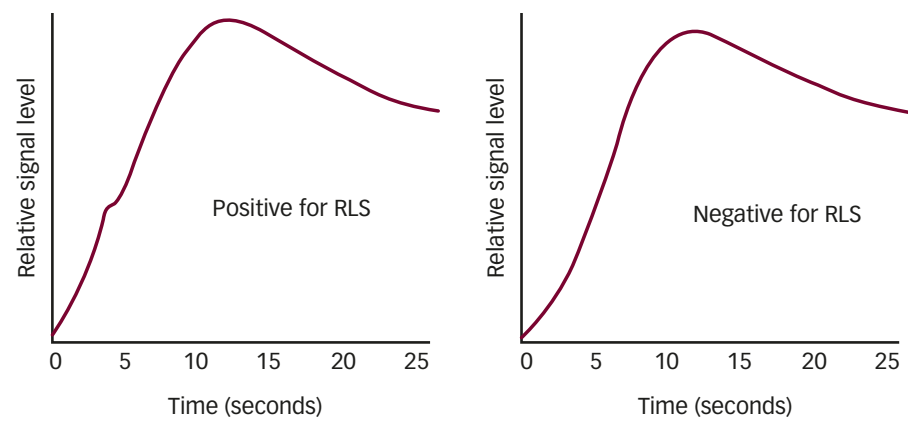

$R L S=$ right-to-left shunting

Valsalva recommended that airway pressure be held at $40 \mathrm{mmHg}$ for a minimum of 10 seconds and then abruptly released. ${ }^{32}$ Specific changes in blood pressure, rate, and volume of venous blood returning to the heart both during and after the maneuver provide invaluable data that can be used in determining size and extent of a PFO. The timing of administration of the contrast agent (microbubbles in saline or saline/blood mixture or dye) with the Valsalva release is also important to properly control so that the contrast agent will be available within the right atrium at the time of the temporary opening of the PFO (Figure 3). ${ }^{33}$

A study conducted in 2013 evaluated the relevance of a performed Valsalva on a TEE compared with TTE when testing for a PFO. Of 108 patients, 48 (44\%) were judged to have PFOs by TEE and/or TTE. In 36 patients ( $33 \%$ total, $75 \%$ of those with PFOs), microbubbles were observed both by TEE and TTE, in seven patients only during TTE, and in five patients only during TEE. In patients who were able to successfully perform the Valsalva maneuver during TEE, 22 PFOs were detected, and two shunts ( $9 \%$ ) were missed. Patients who were unable to perform the maneuver presented with 26 PFOs, and five missed shunts (19\%). The research concluded that an adequate valsalva maneuver is crucial for diagnosis of PFO. ${ }^{34}$

Technology Assessment Scales

Transthoracic Echo and Transesophageal Echocardiography-Bubble Study

Currently, there is no widespread accepted grading mechanism for assessing the degree of a RLS from a PFO. Both transthoracic echocardiography and
TEE use a method of assessing PFO via echocardiography or ultrasound and an injected contrast agent. The contrast study known as "Bubble Study" focuses on the number of bubbles seen in a single still frame within the left atrium. The more bubbles that pass through to the LA, presumably, the larger the shunt is (see Table 1).35,36

\section{Transcranial Doppler-Embolic Strikes}

The Spencer algorithm is a method of grading PFO presence while using the TCD method of sonographic imaging. In this grading scale, a contrast agent is injected and embolic tracks or strikes are measured and recorded when they pass through to the left atrium. Again, the greater the number of bubbles passing through, the greater the perceived significance of a PFO (see Table 2). ${ }^{37}$

\section{ICG Dye Dilution Curves-Peak Spectral Analysis}

With the ICG Dye Dilution curve method of assessing PFO, ICG dye is injected and its appearance rate at the site of the ear is monitored for one or two peaks. One peak indicates no shunting, while two peaks suggest that a portion of dye found a secondary pathway (shunt) that avoided traversing the pulmonary system. ${ }^{31}$

\section{Conclusion}

The established correlation between PFO and a series of clinical manifestations such as stroke/TIA, migraine, OSA, DCl, and others has been documented in a multitude of studies and reports. PFO may be a significant health factor and PFO status should be considered as a potential contributing etiology for these conditions when developing a diagnosis and treatment plan.

There are multiple methodologies now available to assist in the detection of a PFO. It is important for the clinician to understand the various procedures such as TTE, TEE, TCD, and dye dilution curve testing currently being utilized to detect (and in some cases assess the size of) a RLS associated with a PFO. It is important to note that there are some limitations among these varying procedures that could impact the sensitivity and accuracy during interpretation of the results.

A properly performed Valsalva maneuver to open a potential PFO is an important step during any assessment of cardiac shunt as the data it provides is pertinent in measuring the size and volume of shunting that occurs. Testing methods that do not implement this critical component severely reduce the effectiveness of properly identifying PFO.

CLOSURE 1 - Evaluation of the StarFlex Septal Closure System in patients with stroke and/or transischemic attack due to presumed paradoxical embolism through a patent foramen ovale.

RESPECT - Randomized evaluation of recurrent stroke comparing PFO closure to established current standard of care.

PC TRIAL - Comparing percutaneous Closure of PFO using the Amplatzer PFO Occluder with Medical Treatment in patients with cryptogenic embolism.

PREMIUM Migraine Trial - Prospective, Randomized Investigation to Evaluate Incidence of Headache Reduction in Subjects with Migraine and PFO using Amplatzer PFO Occluder to Medical Management. 
1. Hasen $A$, Anjum $P$, Ajmal MR, Patent foramen ovale—clinical significance, JIACM, 2004;5:339-44

2. Hagen P, Scholz D, Edwards W, Incidence and size of patent foramen ovale during the first 10 decades of life: an autopsy study of 965 normal hearts, Mayo Clin Proc, 1984:59:17-20.

3. Kerut EK, Norfleet WT, Plotnick GD, Giles TD, Patent foramen ovale: a review of associated conditions and the impact of physiological size, J Am Coll Cardiol, 2001;38:427-9.

4. Schuchlenz HW, Wolfgang W, Beitzke A, et al., Transesophageal echocardiography for quantifying size of patent foramen ovale in patients with cryptogenic cerebrovascular events, Stroke, 2002;33:293-6.

5. Sacco RL, Ellenberg JH, Mohr JP, et al., Infarcts of undetermined cause: the NINCDS Stroke Data Bank, Ann Neurol 1989;25:382-90.

6. Kim D, Saver J, Patent foramen ovale and stroke: what we do and don't know, Rev Neurol Dis, 2005:2:1-7.

7. Khan AR, Bin Abdulhak AA, Sheikh MA, et al., Device closure of patent foramen ovale versus medical therapy in cryptogenic stroke, JACC Cardiovasc Interv, 2013;6:1316-23.

8. Kent DM, Thaler DE, RoPE Study Investigators, The Risk of Paradoxical Embolism (RoPE) Study: developing risk models for application to ongoing randomized trials of percutaneous patent foramen ovale closure for cryptogenic stroke, Trials, 2011:12:185-94.

9. Morandi E, Anzola GP, Angeli S, et al., Transcatheter closure of patent foramen ovale: a new migraine treatment? J Interv Cardiol, 2003;16:39-41.

10. Schwerzmann $M$, Wiher $S$, Nedeltchev $K$, et al., Percutaneous closure of patent foramen ovale reduces the frequency of migraine attacks, Neurology, 2004;62:1399-401.

11. Reisman M, Christofferson RD, Jesurum J, et al., Migraine headache relief after transcatheter closure of patent foramen ovale, J Am Coll Cardiol, 2005;45:493-5.

12. Tucker ME, Another PFO closure study yields mixed migraine results, Medscape Medical News, June 17, 2015. Available at: www.medscape.com/viewarticle/847080

13. Agnoletti $G$, Iserin $L$, Lafont $A$, et al., Obstructive sleep apnea and patent foramen ovale: successful treatment of symptoms by percutaneous foramen ovale closure, I Interv Cardiol, 2005; 18:393-5.

14. Johansson MC, Eriksson P, Peker Y, et al., The influence of patent foramen ovale on oxygen desaturation in obstructive sleep apnea, Eur Respir J, 2007;29:149-55.

15. Torti SR, Billinger M, Schwerzmann M, et al., Risk of decompression illness among 230 divers in relation to the presence and size of patent foramen ovale, Eur Heart J, 2004;25:1014-20.

16. Purandare N, Burns A, Daly KJ, et al., Cerebral emboli as a potential cause of alzheimer's disease and vascular dementia: case-control study, BMJ, 2006;332:1119-24.

17. Purandare $N$, Welsh $S$, Hutchinson $S$, et al., Cerebral emboli and paradoxical embolisation in dementia: a pilot study, Int I Geriatr Psychiatry, 2005;20:12-6.

18. Desimone $\mathrm{CV}$, Friedman PA, Noheria $\mathrm{A}$, et al., Stroke or transient ischemic attack in patients with transvenous pacemaker or defibrillator and echocardiographically detected patent foramen ovale, Circulation, 2013;1433-41.

19. Pigot B, Kirkham D, Eyrolles $L$, et al., Preventive closure of a patent foramen ovale before total hip replacement, $B r J$ Anaesth, 2009;102:888-9.

20. Mirski MA, Lele AV, Fitzsimmons L, Toung TJK, Diagnosis and treatment of vascular air embolism, Anesthesiology, 2007:106:164-77.

21. Vijay SK, Toeh $H$, Chan B, Diagnosis of a patent foramen ovale JACC CardiovasC Imaging, 2010;3:1084.

22. Mojadidi MK, Bogush N, Caceres JD, et al., Diagnostic accuracy of transesophageal echocardiogram for the detection of patent foramen ovale: a meta-analysis, Echocardiography 2014;31:752-8.

23. Di Tullio M, Sacco RL, Venkestasubramanian N, et al., Comparison diagnostic techniques for the detection of a paten foramen ovale in stroke patients, Stroke, 1993;24:1020-4.

24. Nedelltchev K, Mattle HP, Contrast-enhanced transcranial Doppler ultrasound for diagnosis of patent foramen ovale in stroke patients, Front Neurol Neurosci, 2006;21:206-15.

25. Karltunen $\mathrm{V}$, Ventilä $\mathrm{M}$, Hillbom $\mathrm{M}$, et al., Dye dilution and oximetry for detection of patent foramen ovale, Acta Neurol Scand,
1998;4:231-6

26. Billinger $M$, Schwerzmann $M$, Rutishauser $W$, et al., Patent foramen ovale screening by ear oximetry in divers, $A m$ Cardiol, 2013:111:286-90.

27. Karttunen $\mathrm{V}$, Ventilä $\mathrm{M}$, Ikäheimo $\mathrm{M}$, et al., Ear oximetry: a noninvasive method for detection of patent foramen ovale, Stroke, 2001;32:448-53

28. Carter SA, Swan HJC, Wood EH, Time and concentration components of indicator-dilution curves recorded following central injections of dye in normal human subjects, Circulation, 1959:19:430-9.

29. Banas JS Jr, Meister SG, Gazzaniga AB, et al., A simple technique for detecting small defects of the atrial septum, Am J Cardiol, 1971;28:467-71.

30. Victorica $\mathrm{BE}$, Gessner $\mathrm{IH}$, A simplified method for quantitating eft-to-right shunts from arterial dilution curves, Circulation, 1975;51:530-4.

31. Hemodynamic valsalva simulation created with JSim 2.14 Available at: http://www.physiome.org/ (accessed October 9 2015).

32. Jellinek $E H$, The Valsalva manoeuvre and Antonio Valsalva (1666-1723), J R Soc Med, 2006;99:448-51.

33. Cardiox Corporation. Test Results Showing Two Peak Positive Shunt and One Peak Negative Shunt. Data On File. 2015

34. Rodriques AC, Picard MH, Carbone A, et al., Importance of adequately performed Valsalva maneuver to detect formaen ovale during transesophageal echocardiography, J AT SOC Echocardiogr, 2013;26:1337-43.

35. Braun MU, Fassbender D, Schoen SP, et al., Transcatheter closure of a patent foramen ovale in patients with cerebral ischemia, J Am Coll Cardiol, 2002:39:2019-25.

36. Windecker S, Wahl A, Nedeltchev K, et al., Comparison of medical treatment with percutaneous closure of patent foramen oval in patients with cryptogenic stroke, J Am Coll Cardiol, 2004;44:750-8.

37. Spencer MP, Moehring MA, Jerurum J, et al., Power m-mode transcranial Doppler for diagnosis of patent foramen ovale and assessing transcatheter closure, J Neuroimaging 2004:14:342-9. 